\title{
BMJ Open Association of low-density lipoprotein cholesterol within the normal range and NAFLD in the non-obese Chinese population: a cross-sectional and longitudinal study
}

\author{
Dan-Qin Sun, ${ }^{1}$ Sheng-Jie Wu, ${ }^{2}$ Wen-Yue Liu, ${ }^{3}$ Li-Ren Wang, ${ }^{4,5}$ Yi-Ran Chen, ${ }^{4,5}$ \\ Dong-Chu Zhang, ${ }^{6}$ Martin Braddock, ${ }^{7}$ Ke-Qing Shi, ${ }^{4,8}$ Dan Song, ${ }^{1}$ \\ Ming-Hua Zheng ${ }^{4,8}$
}

To cite: Sun D-Q, Wu S-J, Liu W-Y, et al. Association of low-density lipoprotein cholesterol within the normal range and NAFLD in the nonobese Chinese population: a cross-sectional and longitudinal study. BMJ Open 2016;6:e013781. doi:10.1136/bmjopen-2016013781

- Prepublication history and additional material is available. To view please visit the journal (http://dx.doi.org/ 10.1136/bmjopen-2016013781)

D-QS, S-JW and W-YL are Co-first author.

Received 6 August 2016 Revised 17 October 2016 Accepted 9 November 2016

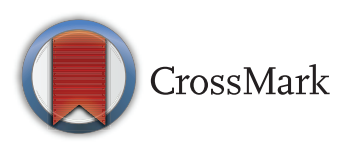

For numbered affiliations see end of article.

Correspondence to

Professor Ming-Hua Zheng; zhengmh@wmu.edu.cn; and Dr Dan Song,

707352346@qq.com

\section{ABSTRACT}

Objectives: The relationship between normal low-density lipoprotein cholesterol (LDL-c) levels and non-alcoholic fatty liver disease (NAFLD) in non-obese individuals remains unclear. We aimed to investigate the precise prevalence and incidence of NAFLD within the normal LDL-c range in non-obese individuals.

Design: Cross-sectional and longitudinal study. Setting: Wenzhou Medical Center of Wenzhou People's Hospital from 2010 to 2014.

Participants: 183903 non-obese individuals were enrolled from a cross-sectional population, and a total of 16173 initially NAFLD-free non-obese individuals were included who completed a 5 -year follow-up examination in the longitudinal population.

Results: In our study, NAFLD was defined by ultrasonographic detection of steatosis in the absence of other liver disease. The cross-sectional study showed that at baseline, the prevalence of NAFLD was $13.9 \%$ in non-obese individuals with normal LDL-c levels. The prospective study demonstrated that NAFLD-free participants developed NAFLD during the 5-year follow-up period, with a cumulative incidence of $14.4 \%$. In addition, the ORs for NAFLD in the cross-sectional population were 1.11 (95\% Cl 1.04 to 1.18$), 1.37$ (95\% Cl 1.27 to 1.47 ) and 1.56 (95\% $\mathrm{Cl} 1.43$ to 1.69$)$, respectively, after adjusting for known confounding variables. The HRs for NAFLD in the longitudinal population were 1.15 ( $95 \% \mathrm{Cl} 0.98$ to 1.36$), 1.32(95 \% \mathrm{Cl} 1.10$ to 1.58 ) and 1.82 ( $95 \% \mathrm{Cl} 1.47$ to 2.52), compared with Q1. Individuals with higher LDL-c level within the normal range had an increased cumulative incidence rate of NAFLD in non-obese individuals.

Conclusions: NAFLD is prevalent in the non-obese Chinese population. Furthermore, this is the first study to demonstrate that increased normal LDL-C levels are independently associated with an elevated risk of NAFLD in non-obese individuals.

\section{Strengths and limitations of this study}

- This is the first and largest study to investigate the relationship between low-density lipoprotein cholesterol within the normal range and nonalcoholic fatty liver disease (NAFLD) in a nonobese Chinese population.

- Stratified and subgroup analysis was used in this study to gain a deep understanding of the relationship.

- The main limitation of this study did not allow for the examination of insulin levels and insulin resistance, although insulin resistance may be closely associated with NAFLD in non-obese individuals.

\section{INTRODUCTION}

Non-alcoholic fatty liver disease (NAFLD) results from hepatic fat accumulation in the absence of quantities of alcohol and any secondary cause and plays an important role in the development of the metabolic syndrome. $^{1-4}$ It is the most common chronic liver disease in Western countries, affecting $31 \%$ of the general population in South America, and recently one meta-analysis reports that it is the relatively high prevalence of NAFLD found in the Asian population $(27 \%) .^{2}{ }^{5} 6$ The prevalence rate of NAFLD in the general population of China varies from $24.77 \%$ to $43.91 \%$ in recent years. ${ }^{6} 7$ NAFLD is recognised as a major cause of liver-related morbidity and mortality, where the mortality accrues from cirrhosis. Furthermore, NAFLD also can increase the risk of cardiovascular disease, type 2 diabetes and chronic kidney disease..$^{8-10}$ 
Obesity, as classified by the body mass index (BMI), has become a worldwide concern reaching epidemic proportions. In the USA, obesity is defined by a BMI of more than $30 \mathrm{~kg} / \mathrm{m}^{2}$. Although BMI and waist circumference can best predict some metabolic disorders, only 2-3\% of Asians are classified as obese by current Western criteria. ${ }^{11}$ Therefore, this cut-off is not universal, and different classifications exist based on racial phenotypic characterisations. The recommended BMI cut-off values for Asians for overweight are $23-25 \mathrm{~kg} / \mathrm{m}^{2}$, and for obesity more than $25 \mathrm{~kg} / \mathrm{m}^{2}$, according to the new BMI criteria for Asians by the regional office for Western Pacific Region of WHO. ${ }^{12-14}$

Obesity is a well-known risk factor for the development of NAFLD, and NAFLD is now recognised as one of the most prevalent manifestations of obesity-related metabolic syndrome (MS).$^{15}$ However, growing evidence has shown that the prevalence rate of NAFLD in populations who are not obese is not uncommon. This may be the case especially in Asian countries, in which the people are generally less obese than those in Western countries and the prevalence of NAFLD is increasing with time. ${ }^{16}$ Studies have shown that about $15-21 \%$ of the Asian patients with NAFLD are non-obese; the proportion of non-obesity in Asians with NAFLD ranged between $11 \%$ in Taiwan, ${ }^{17} 24 \%$ in Korea ${ }^{18}$ and $75 \%$ in India. ${ }^{19}$

Dyslipidaemia is a co-morbidity in the setting of NAFLD and results in hypertriglyceridaemia, reductions in high-density lipoprotein cholesterol (HDL-c), an increase in the size of very low-density lipoprotein and low-density lipoprotein cholesterol (LDL-c).$^{20}$ Moreover, an increasing number of studies have proved that LDL-c is associated with NAFLD and the development of nonalcoholic steatohepatitis (NASH). ${ }^{21-23}$ Meanwhile, we have recently proposed that increased levels of LDL-c within the normal range may play a significant role in the prevalence and incidence of NAFLD. ${ }^{24}$ To gain a deep understanding of the relationship between NAFLD and normal LDL-c in non-obese patients, we conducted our analyses based on a large general cross-sectional and prospective longitudinal Chinese population.

\section{METHODS}

\section{Study design and population}

The participants in the cross-sectional and longitudinal studies were individuals who underwent a health examination in Wenzhou Medical Center of Wenzhou People's Hospital from January 2010 to December 2014. The two studies initially enrolled 372254 participants (339 101 in the cross-sectional group and 33153 in the longitudinal group), of which 200076 eligible individuals remained. Since not all individuals met our criteria, the cross-sectional population only consisted of 183903 populations. The longitudinal population was based on a prospective study and conducted from 33153 initially NAFLD-free individuals. Finally, a total of 16173 initially NAFLD-free non-obese individuals were included, who completed the 5-year follow-up examination. Individuals were excluded if they reported excess alcohol consumption (>140 g/week for men and $>70 \mathrm{~g} /$ week for women), or had a history of viral hepatitis, autoimmune hepatitis or other known causes of chronic liver disease; a BMI of $\geq 25 \mathrm{~kg} / \mathrm{m}^{2}$; an LDL-c of $>3.12 \mathrm{mmol} / \mathrm{L}$; were taking antihypertensive agents, antidiabetic agents or lipid-lowing agents; and were lost to follow-up or their data were missing.

Verbal informed consent was obtained from each participant before their participation in the study. The personal information of participants was erased and replaced by the health examination number. The research protocol of the study was approved by the ethics committee of Wenzhou People's Hospital.

\section{Diagnosis of NAFLD by ultrasonography}

The ultrasound criteria for the diagnosis of fatty liver were based on those suggested by the Chinese Liver Disease Association. ${ }^{25}$ In general, NAFLD was defined as diffuse enhancement of near-field echo in the hepatic region (stronger than in the kidney and spleen region) and gradual attenuation of the far-field echo, if combined with any of the following: unclear display of intrahepatic lacuna structure; mild-to-moderate hepatomegaly with a round and blunt border; a reduction in the blood flow signal, but the distribution of blood flow is normal; and unclear or non-intact display of envelop of the right liver lobe and diaphragm.

\section{Definition of metabolic syndrome}

Metabolic syndrome represents a cluster of physiological and anthropometric abnormalities, requiring $\geq 3$ of the following five factors: (1) waist circumference $>90 \mathrm{~cm}$ for men, and $>80 \mathrm{~cm}$ for women and/or BMI $>25 \mathrm{~kg} / \mathrm{m}^{2}$ in both genders; (2) serum triglyceride (TG) $\geq 1.7 \mathrm{mmol} / \mathrm{L}$; (3) HDL-c $<1.03 \mathrm{mmol} / \mathrm{L}$ for men, $<1.29 \mathrm{mmol} / \mathrm{L}$ for women; (4) systolic blood pressure $\geq 130 \mathrm{~mm} \mathrm{Hg}$ or diastolic blood pressure $\geq 85 \mathrm{~mm} \mathrm{Hg}$ or previously diagnosed; and (5) fasting glucose $\geq 5.6 \mathrm{mmol} / \mathrm{L}$ or previously diagnosed type 2 diabetes.

\section{Data collection}

Baseline clinical examinations were performed as described previously. ${ }^{26}$ In brief, medical history and a health habit inventory were taken by a physician. BMI $\left(\mathrm{kg} / \mathrm{m}^{2}\right)$, used as an index of body fat, was calculated as weight in kilograms divided by height in $\mathrm{m}^{2}$. Blood pressure was measured using an automated sphygmomanometer with the participant in a quiet environment and in a sitting position. The biochemical measurements included albumin (ALB), alanine aminotransferase (ALT), aspartate aminotransferase (AST), fasting plasma glucose (FPG), blood urea nitrogen (BUN), creatinine (Cr), uric acid (UA), total cholesterol (TC), TG, HDL-c, and LDL-c. All values were measured by an automated analyser (Abbott AxSYM) using standard methods. 
Follow-up and outcome evaluations

The follow-up evaluations were performed annually during the observation period. The procedures for follow-up evaluation were the same as those used at baseline. Hepatic ultrasonic examinations were performed in a blinded manner (as at baseline) to determine the incidence of NAFLD. Blood samples were analysed in the same laboratories at baseline and for measurements at follow-up.

\section{Statistical analysis}

In order to derive a deeper understanding of the relationship between a normal range of LDL-c level and the prevalence of NAFLD in the non-obese population, all participants were classified into four groups by quartiles statistically. Quartiles in the cross-sectional and longitudinal populations were categorised separately as follows: Q1: $\leq 1.92 \mathrm{mmol} / \mathrm{L}$; Q2: 1.93-2.27 mmol/L; Q3: 2.28$2.61 \mathrm{mmol} / \mathrm{L}$; and Q4: 2.62-3.12 $\mathrm{mmol} / \mathrm{L}$.

In the cross-sectional non-obese population, the ORs and $95 \%$ CIs for NAFLD were calculated after adjusting for known confounding variables across each quartile of LDL-c concentration using multivariate logistic regression analysis. HRs based on Cox's proportional hazards regression and $95 \%$ CIs were determined in the longitudinal population analysis. The Kaplan-Meier analysis was applied to calculate the cumulative hazard of NAFLD during the follow-up. Multivariable models included sex, age, BMI, FPG, ALB, ALT, AST, BUN, Cr, TC, TG, HDL-c, SBP, DBP and UA.

Statistical analyses were conducted using the SPSS V.18.0 (SPSS, Chicago, Illinois, USA).

Continuous variables were summarised as mean \pm SD, and categorical variables were displayed as counts or percentages $(\%)$. The characteristics of the study population according to LDL-c quartiles were compared using a one-way analysis of variance (ANOVA) for continuous variables and $\chi^{2}$-test for categorical variables. All $\mathrm{p}$ values are two sided, and a $\mathrm{p}$ value of $<0.05$ (two tailed) was considered statistically significant.

\section{RESULTS}

\section{Characteristics of study participants}

A total of 372254 participants were initially enrolled into the study, of which 200076 eligible individuals remained (figure 1). In the cross-sectional population, 183903 non-obese individuals (91 260 men and 92643 women) were enrolled, of which 25503 (19 796 men and 5707 women) fulfilled the diagnostic criteria for

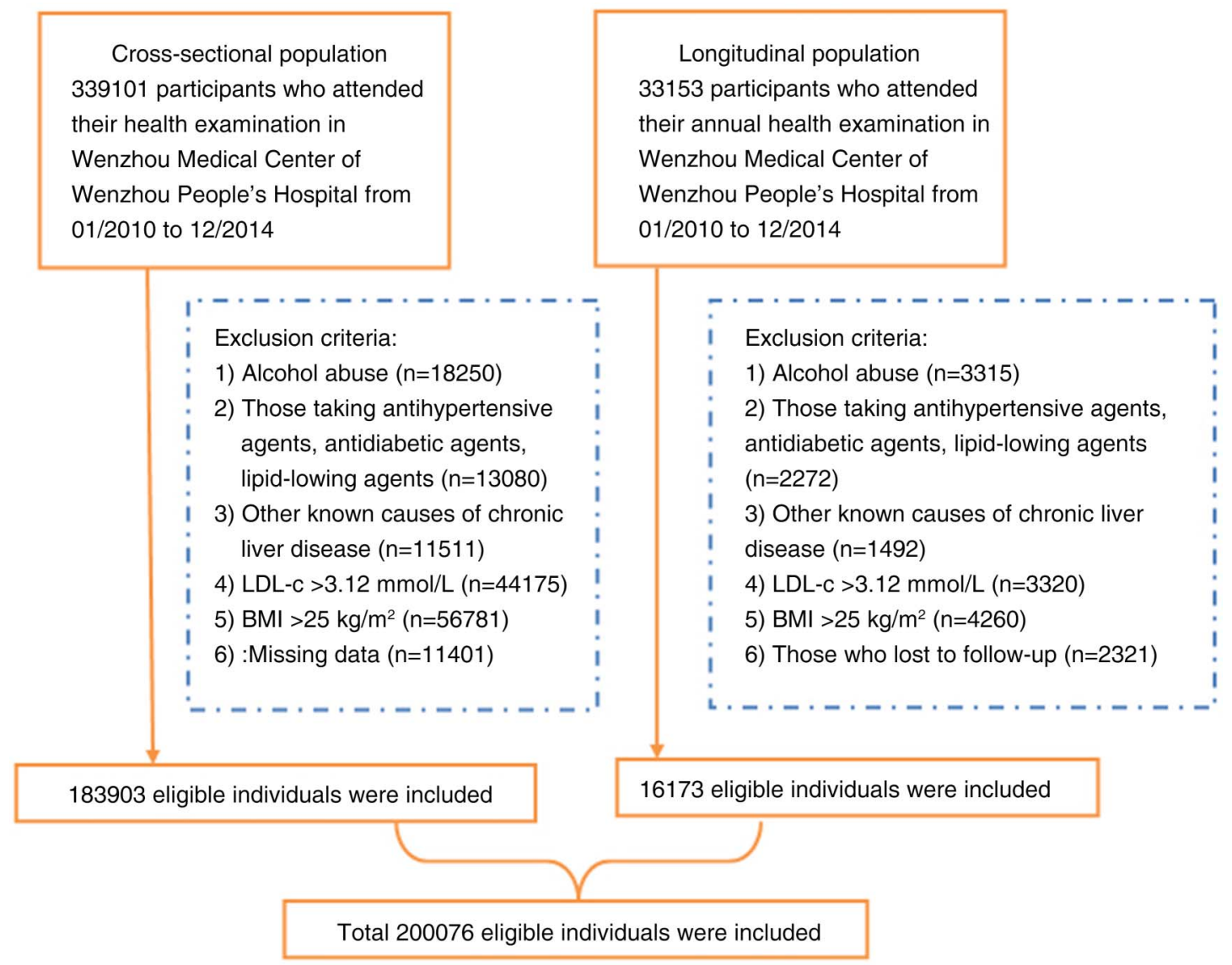

Figure 1 Study flow diagram. A total of 339101 participants were enrolled initially, while 183903 non-obese participants were included in the cross-sectional population. A total of 33153 participants were enrolled initially, while 16173 non-obese participants were included in the longitudinal population. 
NAFLD. The individuals with NAFLD were older and had higher BMI, SBP, DBP, FPG, ALB, ALT, AST, BUN, LDL-c, Cr, TC, TG and UA. The prevalence of NAFLD in men was higher than that in women. Furthermore, the NAFLD group had significantly lower HDL-c levels. At baseline, a total of 16173 initially NAFLD-free nonobese individuals were included, which completed the 5-year follow-up examination. Of the 16173 eligible participants, 2322 non-obese individuals developed into populations with NAFLD, with higher LDL-c than that measured in normal individuals $(2.40 \pm 0.45$ vs $2.24 \pm 0.46$, $\mathrm{p}<0.001)$. The baseline characteristics of participants in cross-sectional and longitudinal non-obese populations are showed in tables 1 and 2, respectively.

\section{High LDL-c though within the normal range related to prevalence of NAFLD in non-obese Chinese}

As shown in tables 1 and 2, non-obese individuals with NAFLD have a higher LDL-c than those without NAFLD. The prevalence of NAFLD in the cross-sectional study was significantly higher in the participants with higher LDL-c than in those with lower LDL-c. It is known that NAFLD is intimately linked with MS and its components. However, it is unclear whether non-obese patients with normal LDL-c levels show an association with NAFLD. Our study investigated the prevalence rate of NAFLD in a subgroup analysis with different groups of individuals with a normal LDL-c level. The subgroup analysis included hypertriglyceridaemia $(\mathrm{TG}<1.7 \mathrm{mmol} / \mathrm{L})$, low HDL-c $(<1.03 \mathrm{mmol} / \mathrm{L})$, blood pressure $(\mathrm{BP}<130 /$ $85 \mathrm{~mm} \mathrm{Hg})$ or FPG $(<5.6 \mathrm{mmol} / \mathrm{L})$ and non-MS. The prevalence in most subgroups showed a linear trend increasing from Q1 to Q4 under normal metabolic conditions (figure 2).
To further understand the relationship between LDL-c level and the prevalence of NAFLD, the OR for NAFLD was calculated after adjusting for confounding variables. Using $\mathrm{Q} 1$ as a reference, the OR for NAFLD was 1.39 (95\% CI 1.33 to 1.45$), 2.13(95 \%$ CI 2.04 to 2.22$)$ and 3.05 (95\% CI 2.93 to 3.18) for Q2, Q3 and Q4, respectively, in model 1 . When analysed by the fully adjusted model (model 3), the relationship between LDL-c and NAFLD remained statistically significant in Q2, Q3 and Q4 with ORs of 1.11 (95\% CI 1.04 to 1.18), 1.37 (95\% CI 1.27 to 1.47 ) and 1.56 (95\% CI 1.43 to 1.69 ), respectively (table 3 ). These results suggest that non-obese Chinese patients with higher LDL-c levels are more likely to develop NAFLD than patients with lower LDL-c levels.

Figure 3 shows forest plots of OR for quartiles of LDL-c in the cross-sectional non-obese population. A stratified analysis of risk factors for normal and abnormal metabolic conditions all showed a successive increase in OR from Q1 to Q4. However, a higher OR for NAFLD was observed in participants with non-MS or its normal components than those with abnormal conditions (figure 3).

High LDL-c though within the normal range predicts the incidence risk of NAFLD in non-obese Chinese population To investigate if an increased level of LDL-c within the normal range may play a role in the development of NAFLD in the non-obese Chinese population, a longitudinal population was included. A total of 2322 participants developed NAFLD during the follow-up study. The incidence rate of NAFLD in non-MS and its subgroups showed linear increasing trends from Q1 to Q4 (figure 2). The Kaplan-Meier analysis showed that the cumulative

Table 1 Baseline characteristics of cross-sectional non-obese population

\begin{tabular}{|c|c|c|c|}
\hline Characteristics & Without NAFLD & With NAFLD & p Value \\
\hline Male (female) & 71464 (86 936) & $19796(5707)$ & \\
\hline Age, years & $40.04 \pm 13.91$ & $46.81 \pm 13.24$ & $<0.001$ \\
\hline BMI, $\mathrm{kg} / \mathrm{m}^{2}$ & $21.12 \pm 2.07$ & $23.37 \pm 1.24$ & $<0.001$ \\
\hline $\mathrm{SBP}, \mathrm{mm} \mathrm{Hg}$ & $118.26 \pm 15.60$ & $128.25 \pm 15.63$ & $<0.001$ \\
\hline $\mathrm{DBP}, \mathrm{mm} \mathrm{Hg}$ & $72.06 \pm 9.97$ & $78.77 \pm 10.37$ & $<0.001$ \\
\hline $\mathrm{FPG}, \mathrm{mmol} / \mathrm{L}$ & $5.08 \pm 0.73$ & $5.59 \pm 1.31$ & $<0.001$ \\
\hline ALB, U/L & $44.51 \pm 2.84$ & $45.02 \pm 2.77$ & $<0.001$ \\
\hline $\mathrm{ALT}, \mathrm{U} / \mathrm{L}$ & $18.82 \pm 17.31$ & $29.85 \pm 19.86$ & $<0.001$ \\
\hline AST, U/L & $22.15 \pm 11.12$ & $26.36 \pm 19.86$ & $<0.001$ \\
\hline BUN, mmol/L & $4.37 \pm 1.29$ & $4.67 \pm 3.58$ & $<0.001$ \\
\hline $\mathrm{Cr}, \mathrm{mmol} / \mathrm{L}$ & $77.68 \pm 22.62$ & $84.79 \pm 19.03$ & $<0.001$ \\
\hline $\mathrm{TG}, \mathrm{mmol} / \mathrm{L}$ & $1.18 \pm 0.76$ & $2.28 \pm 1.69$ & $<0.001$ \\
\hline $\mathrm{TC}, \mathrm{mmol} / \mathrm{L}$ & $4.49 \pm 0.73$ & $4.80 \pm 0.78$ & $<0.001$ \\
\hline HDL-c, mmol/L & $1.48 \pm 0.35$ & $1.25 \pm 0.31$ & $<0.001$ \\
\hline $\mathrm{UA}, \mu \mathrm{mol} / \mathrm{L}$ & $273.16 \pm 84.87$ & $345.03 \pm 88.35$ & $<0.001$ \\
\hline LDL-c, $\mu \mathrm{mol} / \mathrm{L}$ & $2.22 \pm 0.47$ & $2.42 \pm 0.45$ & $<0.001$ \\
\hline
\end{tabular}

ALB, albumin; ALT, alanine aminotransferase; AST, aspartate aminotransferase; BMI, body mass index; BUN, blood urea nitrogen; $\mathrm{Cr}$, creatinine; DBP, diastolic blood pressure; FPG, fasting plasma glucose; HDL-c, high-density lipoprotein cholesterol; LDL-c, low-density lipoprotein cholesterol; NAFLD, non-alcoholic fatty liver disease; SBP, systolic blood pressure; TC, total cholesterol; TG, triglyceride; UA, uric acid. 
Table 2 Baseline characteristics of longitudinal non-obese population

\begin{tabular}{|c|c|c|c|}
\hline Characteristics & Without NAFLD & With NAFLD & p Value \\
\hline Male (female) & 7189 (6662) & $1294(1028)$ & \\
\hline Age, years & $42.98 \pm 14.92$ & $44.72 \pm 15.13$ & $<0.001$ \\
\hline $\mathrm{BMI}, \mathrm{kg} / \mathrm{m}^{2}$ & $21.08 \pm 1.99$ & $23.18 \pm 1.34$ & $<0.001$ \\
\hline $\mathrm{SBP}, \mathrm{mm} \mathrm{Hg}$ & $119.49 \pm 16.51$ & $128.09 \pm 16.04$ & $<0.001$ \\
\hline $\mathrm{DBP}, \mathrm{mm} \mathrm{Hg}$ & $71.97 \pm 10.13$ & $77.82 \pm 10.27$ & $<0.001$ \\
\hline $\mathrm{FPG}, \mathrm{mmol} / \mathrm{L}$ & $5.09 \pm 0.73$ & $5.45 \pm 1.01$ & $<0.001$ \\
\hline ALB, U/L & $44.38 \pm 2.70$ & $44.48 \pm 2.78$ & 0.126 \\
\hline $\mathrm{ALT}, \mathrm{U} / \mathrm{L}$ & $18.74 \pm 16.19$ & $26.20 \pm 16.41$ & $<0.001$ \\
\hline AST, U/L & $22.62 \pm 9.56$ & $25.01 \pm 9.17$ & $<0.001$ \\
\hline BUN, mmol/L & $4.56 \pm 1.38$ & $4.60 \pm 1.28$ & 0.195 \\
\hline $\mathrm{Cr}, \mathrm{mmol} / \mathrm{L}$ & $77.25 \pm 26.32$ & $85.82 \pm 20.01$ & $<0.001$ \\
\hline $\mathrm{TG}, \mathrm{mmol} / \mathrm{L}$ & $1.19 \pm 0.73$ & $1.99 \pm 1.46$ & $<0.001$ \\
\hline $\mathrm{TC}, \mathrm{mmol} / \mathrm{L}$ & $4.59 \pm 0.73$ & $4.80 \pm 0.78$ & $<0.001$ \\
\hline HDL-c, mmol/L & $1.49 \pm 0.36$ & $1.30 \pm 0.32$ & $<0.001$ \\
\hline $\mathrm{UA}, \mu \mathrm{mol} / \mathrm{L}$ & $271.87 \pm 83.33$ & $327.18 \pm 85.92$ & $<0.001$ \\
\hline LDL-c, mmol/L & $2.24 \pm 0.46$ & $2.40 \pm 0.45$ & $<0.001$ \\
\hline
\end{tabular}
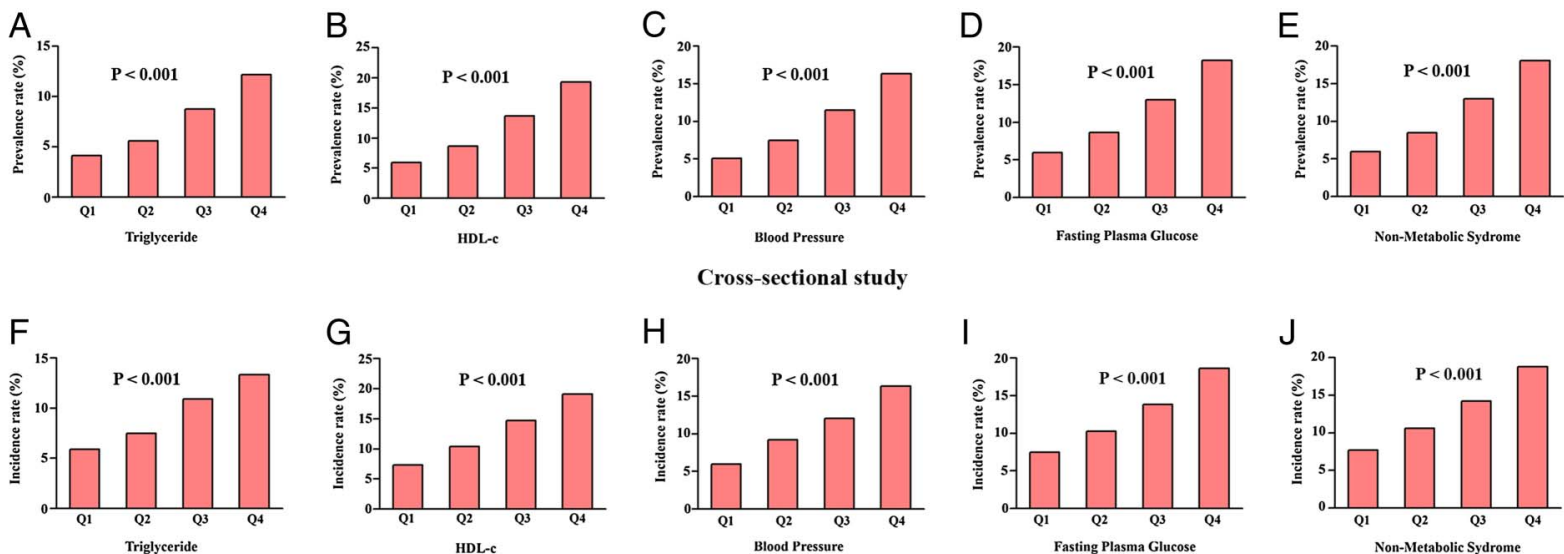

Cross-sectional study
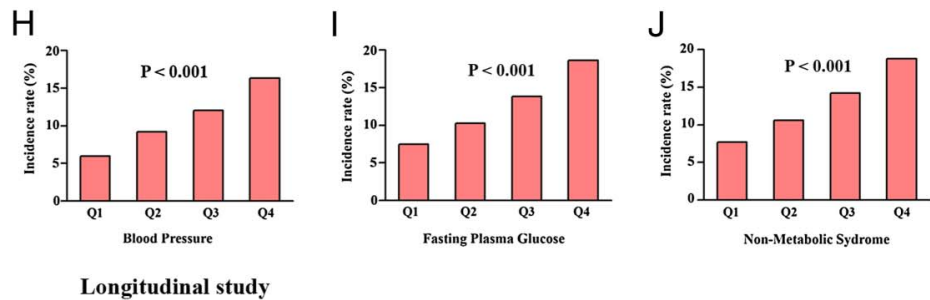

Figure 2 Prevalence and incidence rate of NAFLD in the cross-sectional population and the longitudinal population. The prevalence rate and incidence rate of NAFLD in the subgroups analysis including hypertriglyceridaemia (TG $<1.7 \mathrm{mmol} / \mathrm{L})$, low HDL-c (<1.03 mmol/L), blood pressure (BP $<130 / 85 \mathrm{~mm} \mathrm{Hg})$ and FPG $(<5.6 \mathrm{mmol} / \mathrm{L})$ and non-metabolic syndrome all showed increasing trends with the increases in normal low-density lipoprotein cholesterol levels. BP, blood pressure; FPG, fasting plasma glucose; HDL-c, high-density lipoprotein cholesterol; NAFLD, non-alcoholic fatty liver disease.

incidence of NAFLD increased progressively with increased normal LDL-c level (figure 4).

To estimate HR of each LDL-c level quartile for incident NAFLD, Cox's proportional hazards regression analyses were applied. As shown in table 3, the HR for NAFLD was 1.17 (95\% CI 1.02 to 1.34 ), 1.57 (95\% CI 1.40 to 1.80$)$ and 2.09 (95\% CI 1.85 to 2.36$)$ for Q2, Q3 and $\mathrm{Q} 4$, respectively, in unadjusted model 1. After adjusted for age and gender, the HR was similar in model 1. Using a fully adjusted model (model 3), the relationship between LDL-c and NAFLD remained significant in Q2, Q3 and Q4 with HRs of 1.15 (95\% CI 0.98 to 1.36$), 1.32$ (95\% CI 1.10 to 1.58$)$ and $1.82(95 \%$
CI 1.47 to 2.52), respectively (table 3). Online supplementary figure $1 \mathrm{~A}$ and $1 \mathrm{~B}$ shows the unadjusted and adjusted OR and HR of normal LDL-c levels for NAFLD in the cross-sectional and longitudinal nonobese population, respectively. These results indicate that high LDL-c, though within the normal range, may predict the incidence risk of NAFLD in the non-obese Chinese population.

\section{DISCUSSION}

To the best of our knowledge, this is the first study to investigate the relationship between LDL-c within the 
Table 3 Adjusted OR or HR $(95 \% \mathrm{Cl})$ for non-alcoholic fatty liver disease

\begin{tabular}{|c|c|c|c|}
\hline Quartiles of LDL-c & Model 1 & Model 2 & Model 3 \\
\hline \multicolumn{4}{|c|}{ Cross-sectional non-obese population } \\
\hline Q1 & $1.00(1.00$ to 1.00$)$ & $1.00(1.00$ to 1.00$)$ & $1.00(1.00$ to 1.00$)$ \\
\hline Q2 & 1.39 (1.33 to 1.45$)$ & $1.28(1.22$ to 1.34$)$ & $1.11(1.04$ to 1.18$)$ \\
\hline Q3 & 2.13 (2.04 to 2.22$)$ & $1.78(1.71$ to 1.86$)$ & $1.37(1.27$ to 1.47$)$ \\
\hline Q4 & 3.05 (2.93 to 3.18$)$ & 2.31 (2.22 to 2.41$)$ & $1.56(1.43$ to 1.69$)$ \\
\hline $\mathrm{p}$ Value & $<0.001$ & $<0.001$ & $<0.001$ \\
\hline \multicolumn{4}{|c|}{ Longitudinal non-obese population } \\
\hline Q1 & $1.00(1.00$ to 1.00$)$ & $1.00(1.00$ to 1.00$)$ & $1.00(1.00$ to 1.00$)$ \\
\hline Q2 & 1.17 (1.02 to 1.34$)$ & $1.17(1.02$ to 1.34$)$ & $1.15(0.98$ to 1.36$)$ \\
\hline Q3 & 1.57 (1.40 to 1.80$)$ & 1.57 (1.38 to 1.79$)$ & $1.32(1.10$ to 1.58$)$ \\
\hline Q4 & $2.09(1.85$ to 2.36$)$ & 2.06 (1.82 to 2.33$)$ & 1.82 (1.47 to 2.52$)$ \\
\hline $\mathrm{p}$ Value & $<0.001$ & $<0.001$ & $<0.001$ \\
\hline
\end{tabular}

A

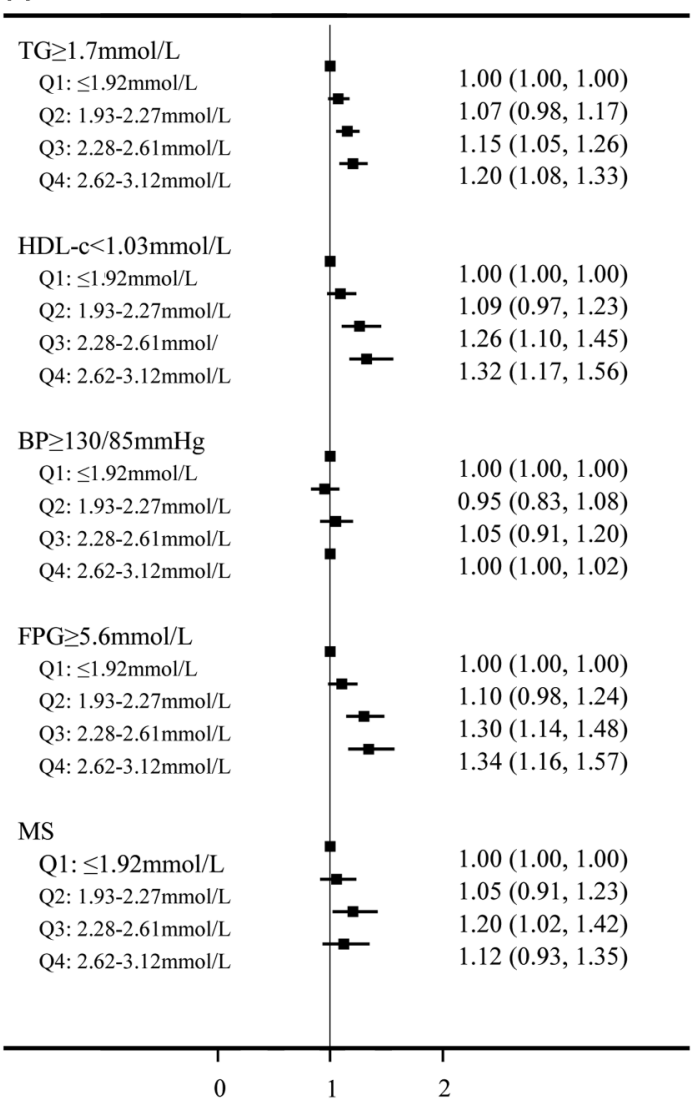

B

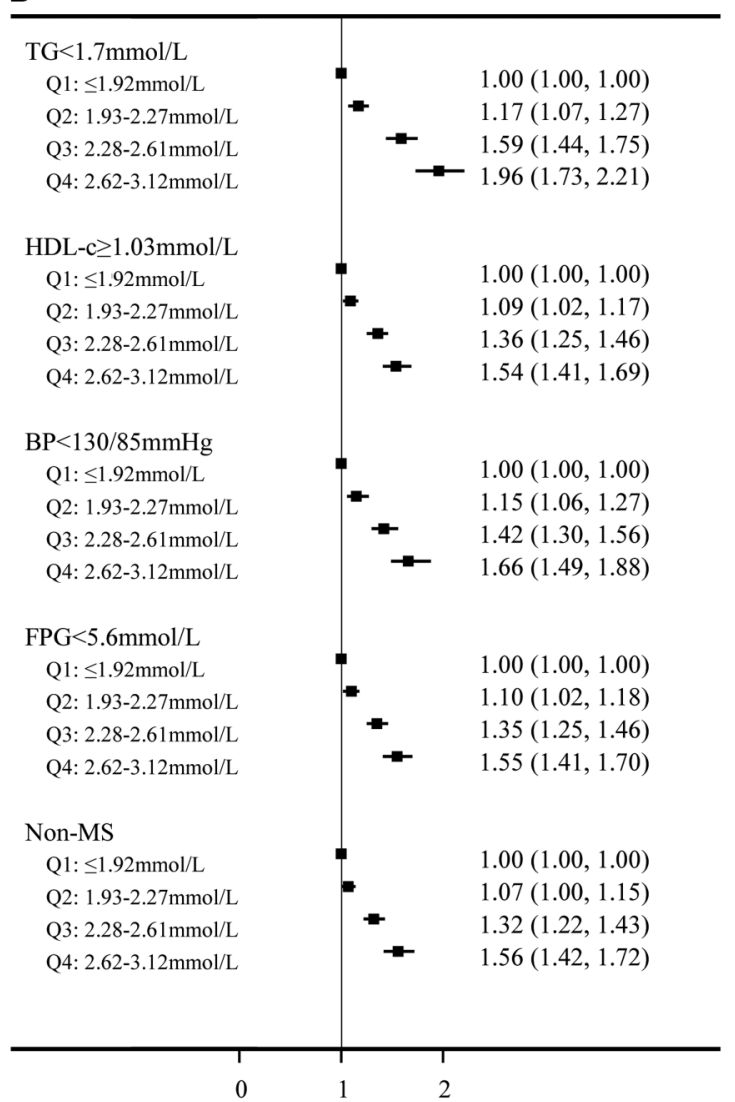

Figure 3 Forest plots of ORs $(95 \% \mathrm{Cl})$ for quartiles of LDL-c in the cross-sectional population. Confounding variables contained age, sex, body mass index, systolic blood pressure, diastolic blood pressure, fasting plasmaglucose, albumin, alanine aminotransferase, aspartate aminotransferase, blood urea nitrogen, creatinine, total cholesterol, triglyceride, high-density lipoprotein cholesterol and uric acid. Increasing trends of OR for NAFLD with the increases in normal LDL-c levels are shown. Q1: $\leq 1.92 \mathrm{mmol} / \mathrm{L}, \mathrm{Q} 2: 1.93-2.27 \mathrm{mmol} / \mathrm{L}, \mathrm{Q} 3:$ : 2.28-2.61 mmol/L and Q4: 2.62-3.12 mmol/L. LDL-c, low-density lipoprotein cholesterol; NAFLD, non-alcoholic fatty liver disease. 


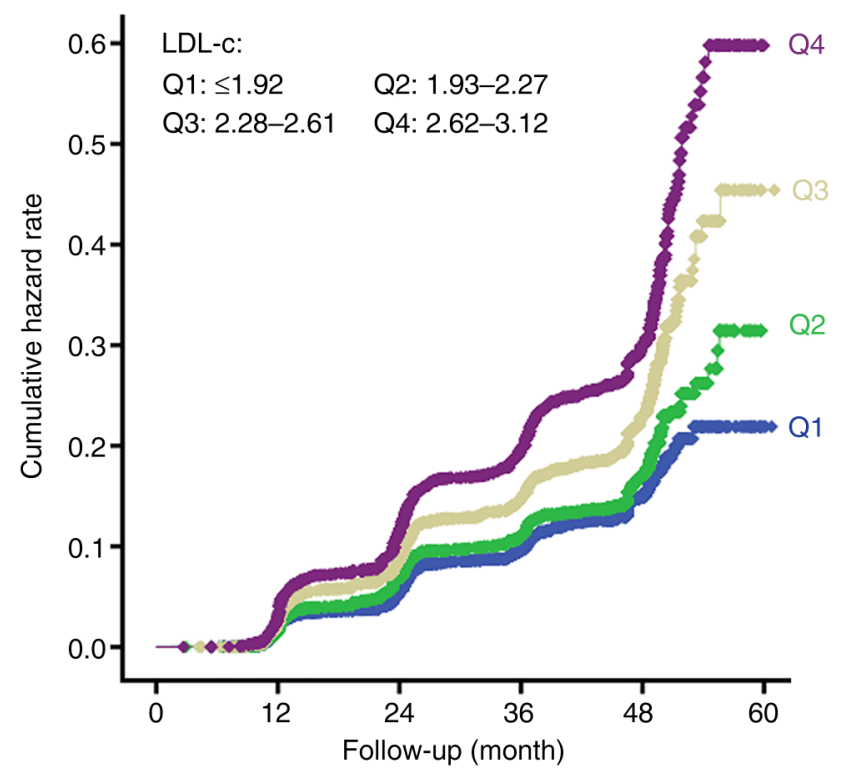

Figure 4 The Kaplan-Meier curves reflecting cumulative incidence rate of NAFLD in the longitudinal non-obese population according to quartiles of normal LDL-c level. Individuals with higher LDL-c level within the normal range had an increased cumulative incidence rate of NAFLD in non-obese individuals. $p$-Value for trend is computed from Cox's analysis. $P_{\text {trend }}<0.001$. LDL-c, low-density lipoprotein cholesterol; NAFLD, non-alcoholic fatty liver disease.

normal range and NAFLD in a non-obese Chinese population. Our cross-sectional study showed that at baseline, the prevalence of NAFLD was $13.9 \%$ in nonobese participants with normal LDL-c levels. The prospective study demonstrated that a substantial proportion of participants developed NAFLD during the 5 -year follow-up period, with a cumulative incidence of $14.4 \%$. Our results showed that NAFLD is prevalent in the non-obese Chinese population enrolled in this study. The follow-up study showed that the number of individuals with NAFLD increased over 5 years. Rapid economic growth and certain lifestyle choices may have contributed to the development of NAFLD in non-obese Chinese people in recent years, and our results are in agreement with previous studies. ${ }^{1627} 28$

However, in previous studies, the relationship between the risk factor LDL-c and NAFLD was not fully described. First, in our cross-sectional study, the prevalence of NAFLD gradually increased as the LDL-c level increased. As NAFLD is closely linked to MS, this may partially explain why an elevated level of LDL-c appears to significantly increase the risk of NAFLD. Besides, we also noticed the association between some non-obese patients with normal metabolic indices and the prevalence of NAFLD. The prevalence rate of NAFLD in our stratified analysis with different groups of individuals with normal LDL-c levels showed a linear trend increasing under normal metabolic conditions. Second, logistic regression analysis further showed that elevated LDL-c level in the normal range made a significant contribution to the risk for NAFLD in non-obese individuals. Third, in our prospective longitudinal non-obese population, high LDL-c though within the normal range appears to increase the incidence risk of NAFLD.

Dyslipidaemia is a well-known risk factor for NAFLD. Especially, hypertriglyceridaemia plays an important role in the presence, development and regression of NAFLD in non-obese individuals. ${ }^{17} 18 \quad 2729$ Excessively high hepatic TG concentrations and hypertriglyceridaemia result from an increased influx of free fatty acids from visceral fat deposits and in the secretion of very lowdensity lipoprotein from the liver. Sinn $e t a b^{30}$ reported that NAFLD is an independent predictor of insulin resistance, irrespective of the number of the metabolic components of MS in non-obese Asian adults. In addition, insulin resistance is associated with the pathogenesis of NAFLD in non-obese individuals, irrespective of the presence of the MS. ${ }^{18}$ Under conditions of insulin resistance, multiple metabolic abnormalities can conspire to increase the secretion of very low-density lipoprotein and increase the proportion of small dense LDL particles. $^{31}$ Therefore, insulin resistance may partially explain why elevation of LDL-c appears to significantly increase the risk of NAFLD in non-obese individuals.

In our study, we wished to demonstrate that the associations between normal LDL-c levels and NAFLD are also observed in patients with normal metabolic indices. The prevalence rate of NAFLD in our subgroup analysis increased under normal metabolic conditions. Moreover, the relationship between LDL-c and NAFLD remained statistically significant after adjusting for features of MS and other known confounding variables in multivariate logistic and Cox's proportional hazards regression analysis. The Framingham Heart Study had suggested that a fatty liver is associated with dyslipidemia and dysglycaemia and is independent of fat deposits, including visceral adipose tissue. ${ }^{32}$ The MESA study indicated that primary lipoprotein abnormalities resulting in hepatic TG over production or impaired secretion, independent of insulin resistance may be responsible for NAFLD. ${ }^{33}$ In addition, low-density lipoprotein receptorrelated protein 6 (LRP6) may explain some mechanisms of associating LDL-c with NAFLD. LRP6, a member of the low-density lipoprotein receptor family, plays an important role in lipid homeostasis, glucose metabolism and atherosclerosis. Impaired function of LRP6 may drive increased serum TG levels and serum LDL, and enhanced LDL deposition in the liver which are major risk factors for NAFLD, atherosclerosis and together constitute the major components of MS. ${ }^{34} 35$

The interpretation of this study has some limitations. The main limitation is a lack of anthropometric parameters regarding central obesity, lifestyle and dietary factors. In addition, this study may be considered limited in that NAFLD was diagnosed by ultrasonographic methods, which cannot determine the severity of NAFLD. Nevertheless, ultrasonography is widely used in epidemiological surveys of NAFLD because of its safety, economical 
and practical utility. ${ }^{5}$ Third, our initial study design did not allow for the examination of insulin levels and insulin resistance, although insulin resistance may be closely associated with NAFLD in non-obese individuals. ${ }^{30}$

In conclusion, our results showed that NAFLD is prevalent in the non-obese Chinese population, and a substantial proportion of participants developed NAFLD during the 5-year follow-up period. In addition, we conclude that increased normal LDL-c levels are independently associated with an elevated risk of NAFLD in non-obese individuals. Furthermore, LDL-c evaluation and control of LDL-c should be a focal point for health checks in non-obese individuals, to improve the prevention and management of NAFLD.

\section{Author affiliations}

${ }^{1}$ Department of Nephrology, Affiliated Wuxi Second Hospital, Nanjing Medical University, Wuxi, China

${ }^{2}$ Department of Cardiovascular Medicine, The Heart Center, the First Affiliated Hospital of Wenzhou Medical University, Wenzhou, China

${ }^{3}$ Department of Endocrinology, The First Affiliated Hospital of Wenzhou Medical University, Wenzhou, China

${ }^{4}$ Department of Hepatology, Liver Research Center, the First Affiliated Hospital of Wenzhou Medical University, Wenzhou, China

${ }^{5}$ School of the First Clinical Medical Sciences, Wenzhou Medical University, Wenzhou, China

${ }^{6}$ Wenzhou Medical Center, Wenzhou People's Hospital, Wenzhou, China

${ }^{7}$ Global Medicines Development, AstraZeneca R\&D, Alderley Park, UK

${ }^{8}$ Institute of Hepatology, Wenzhou Medical University, Wenzhou, China

Contributors D-QS, S-JW, W-YL and D-CZ MH designed the study. D-QS, W-YL and D-CZ screened and extracted data. L-RW, Y-RC and S-JW did the statistical analyses. K-QS and L-RW prepared figures. D-QS, MB, DS and $\mathrm{M}-\mathrm{HZ}$ reviewed the results, interpreted data and wrote the manuscript. All authors saw and approved the final version of the paper

Funding This work was supported by grants from National Natural Science Foundation of China (81500665), High Level Creative Talents from Department of Public Health in Zhejiang Province and Project of New Century 551 Talent Nurturing in Wenzhou.

Competing interests None declared.

Ethics approval Ethics committee of Wenzhou People's Hospital.

Provenance and peer review Not commissioned; externally peer reviewed.

Data sharing statement Extra data can be accessed via the Dryad data repository at http://datadryad.org/ with the doi:10.5061/dryad.1n6c4

Open Access This is an Open Access article distributed in accordance with the Creative Commons Attribution Non Commercial (CC BY-NC 4.0) license, which permits others to distribute, remix, adapt, build upon this work noncommercially, and license their derivative works on different terms, provided the original work is properly cited and the use is non-commercial. See: http:// creativecommons.org/licenses/by-nc/4.0/

\section{REFERENCES}

1. Sun DQ, Wu SJ, Liu WY, et al. Serum uric acid: a new therapeutic target for nonalcoholic fatty liver disease. Expert Opin Ther Targets 2016;20:375-87.

2. Wu SJ, Zou H, Zhu GQ, et al. Increased levels of systolic blood pressure within the normal range are associated with significantly elevated risks of nonalcoholic fatty liver disease. Medicine (Baltimore) 2015;94:e842.

3. Ballestri S, Zona S, Targher G, et al. Nonalcoholic fatty liver disease is associated with an almost twofold increased risk of incident type 2 diabetes and metabolic syndrome. Evidence from a systematic review and meta-analysis. J Gastroenterol Hepatol 2016;31:936-44.
4. Lonardo A, Ballestri S, Marchesini G, et al. Nonalcoholic fatty liver disease: a precursor of the metabolic syndrome. Dig Liver Dis 2015;47:181-90.

5. Angulo P. Nonalcoholic fatty liver disease. $N$ Engl J Med 2002;346:1221-31.

6. Younossi ZM, Koenig AB, Abdelatif D, et al. Global epidemiology of nonalcoholic fatty liver disease-meta-analytic assessment of prevalence, incidence, and outcomes. Hepatology 2016;64:73-84.

7. Amarapurkar DN, Hashimoto E, Lesmana LA, et al. How common is non-alcoholic fatty liver disease in the Asia-Pacific region and are there local differences? J Gastroenterol Hepatol 2007;22:788-93.

8. Liu H, Lu HY. Nonalcoholic fatty liver disease and cardiovascular disease. World J Gastroenterol 2014;20:8407-15.

9. Musso G, Gambino R, Tabibian JH, et al. Association of non-alcoholic fatty liver disease with chronic kidney disease: a systematic review and meta-analysis. PLoS Med 2014;11:e1001680.

10. Sung KC, Kim SH. Interrelationship between fatty liver and insulin resistance in the development of type 2 diabetes. J Clin Endocrinol Metab 2011;96:1093-7.

11. Deurenberg P, Deurenberg-Yap M, Guricci S. Asians are different from Caucasians and from each other in their body mass index/body fat per cent relationship. Obes Rev 2002;3:141-6.

12. Zimmet $P$, Magliano $D$, Matsuzawa $Y$, et al. The metabolic syndrome: a global public health problem and a new definition. $J$ Atheroscler Thromb 2005;12:295-300.

13. Fan JG, Saibara T, Chitturi S, et al. What are the risk factors and settings for non-alcoholic fatty liver disease in Asia-Pacific? J Gastroenterol Hepatol 2007;22:794-800.

14. Chitturi S, Farrell GC, Hashimoto E, et al. Non-alcoholic fatty liver disease in the Asia-Pacific region: definitions and overview of proposed guidelines. J Gastroenterol Hepatol 2007;22:778-87.

15. Fabbrini E, Sullivan S, Klein S. Obesity and nonalcoholic fatty liver disease: biochemical, metabolic, and clinical implications. Hepatology 2010:51:679-89.

16. Liu CJ. Prevalence and risk factors for non-alcoholic fatty liver disease in Asian people who are not obese. J Gastroenterol Hepato 2012;27:1555-60.

17. Chen $\mathrm{CH}$, Huang $\mathrm{MH}$, Yang JC, et al. Prevalence and risk factors of nonalcoholic fatty liver disease in an adult population of Taiwan: metabolic significance of nonalcoholic fatty liver disease in nonobese adults. J Clin Gastroenterol 2006;40:745-52.

18. Kim HJ, Lee KE, Kim DJ, et al. Metabolic significance of nonalcoholic fatty liver disease in nonobese, nondiabetic adults. Arch Intern Med 2004;164:2169-75.

19. Das K, Mukherjee PS, Ghosh A, et al. Nonobese population in a developing country has a high prevalence of nonalcoholic fatty liver and significant liver disease. Hepatology 2010;51:1593-602.

20. Gaggini M, Morelli M, Buzzigoli E, et al. Non-alcoholic fatty liver disease (NAFLD) and its connection with insulin resistance dyslipidemia, atherosclerosis and coronary heart disease. Nutrients 2013;5:1544-60.

21. Targher G, Bertolini L, Rodella S, et al. NASH predicts plasma inflammatory biomarkers independently of visceral fat in men. Obesity (Silver Spring) 2008;16:1394-9.

22. Imajo $\mathrm{K}$, Hyogo $\mathrm{H}$, Yoneda $\mathrm{M}$, et al. LDL-migration index (LDL-MI), an indicator of small dense low-density lipoprotein (sdLDL), is higher in non-alcoholic steatohepatitis than in non-alcoholic fatty liver: a multicenter cross-sectional study. PLoS One 2014:9:e115403.

23. Norris AL, Steinberger J, Steffen LM, et al. Circulating oxidized LDL and inflammation in extreme pediatric obesity. Obesity (Silver Spring) 2011:19:1415-19.

24. Sun DQ, Liu WY, Wu SJ, et al. Increased levels of low-density lipoprotein cholesterol within the normal range as a risk factor for nonalcoholic fatty liver disease. Oncotarget 2016;7:5728-37.

25. Zeng MD, Fan JG, Lu LG, et al. Guidelines for the diagnosis and treatment of nonalcoholic fatty liver diseases. J Dig Dis 2008:9:108-12.

26. Wu SJ, Zhu GQ, Ye BZ, et al. Association between sex-specific serum uric acid and non-alcoholic fatty liver disease in Chinese adults: a large population-based study. Medicine (Baltimore) 2015;94:e802.

27. $\mathrm{Xu} \mathrm{C}, \mathrm{Yu} \mathrm{C}, \mathrm{Ma} \mathrm{H}$, et al. Prevalence and risk factors for the development of nonalcoholic fatty liver disease in a nonobese Chinese population: the Zhejiang Zhenhai study. Am J Gastroenterol 2013;108:1299-304.

28. Cho HC. Prevalence and factors associated with nonalcoholic fatty liver disease in a nonobese Korean population. Gut Liver 2016;10:117-25.

29. Kim NH, Kim JH, Kim YJ, et al. Clinical and metabolic factors associated with development and regression of nonalcoholic fatty liver disease in nonobese subjects. Liver Int 2014;34:604-11. 
30. Sinn DH, Gwak GY, Park HN, et al. Ultrasonographically detected non-alcoholic fatty liver disease is an independent predictor for identifying patients with insulin resistance in non-obese, non-diabetic middle-aged Asian adults. Am J Gastroenterol 2012;107:561-7.

31. Lewis GF, Murdoch S, Uffelman K, et al. Hepatic lipase mRNA, protein, and plasma enzyme activity is increased in the insulin-resistant, fructose-fed Syrian golden hamster and is partially normalized by the insulin sensitizer rosiglitazone. Diabetes 2004;53:2893-900.

32. Speliotes EK, Massaro JM, Hoffmann U, et al. Fatty liver is associated with dyslipidemia and dysglycemia independent of visceral fat: the Framingham Heart study. Hepatology 2010;51:1979-87.

33. DeFilippis AP, Blaha MJ, Martin SS, et al. Nonalcoholic fatty liver disease and serum lipoproteins: the multi-ethnic study of atherosclerosis. Atherosclerosis 2013;227:429-36.

34. Mani A, Radhakrishnan J, Wang $\mathrm{H}$, et al. LRP6 mutation in a family with early coronary disease and metabolic risk factors. Science 2007;315:1278-82.

35. Go GW, Srivastava R, Hernandez-Ono A, et al. The combined hyperlipidemia caused by impaired Wnt-LRP6 signaling is reversed by Wnt3a rescue. Cell Metab 2014;19:209-20. 